\title{
Polymerization of Acrylamide in Aqueous Medium Initiated with a Redox System of Cysteine and Ammonium Persulfate
}

\author{
Takuji OKaYa, Kenji KIKUCHI, and Yukiko MorII \\ Department of Materials Science, The University of Shiga Prefecture, \\ 2500 Hassaka, Hikone 522, Japan
}

(Received December 24, 1996)

\begin{abstract}
Aqueous polymerization of acrylamide initiated with a redox system of cysteine containing a thiol group and ammonium persulfate was investigated. This is a model of the redox initiator system composed of poly(vinyl alcohol) having a thiol group at one end and ammonium persulfate which polymerized many monomers yielding various block copolymers. The rate of polymerization was proportional to the 0.5 power of the concentrations of cysteine and of ammonium persulfate and 1.0 power of monomer concentration, indicating the initiator system to be a usual free radical polymerization initiator. The activation energy of over-all rate of polymerization was $4 \mathrm{kcal} \mathrm{mol}^{-1}$ indicating a typical redox initiator of the system. Rate constant of the redox decomposition reaction, $k$, was determined as $1.7 \mathrm{~L} \mathrm{~mol}^{-1} \mathrm{~s}^{-1}$. Chain transfer constant of polyacrylamide radical to ammonium persulfate was calculated to be 0.65 .

KEY WORDS Redox Initiator / Thiol / Cysteine / Chain Transfer / Ammonium Persulfate / Acrylamide /
\end{abstract}

Maiti and Palit ${ }^{1}$ reported a redox initiator system of a low molecular weight compound containing a thiol group and an oxidant in the polymerization of methyl methacrylate (MMA) in aqueous medium. In combination with L-cysteine hydrochloride as a reductant, potassium bromate was shown to be the most reactive oxidant among those such as potassium persulfate and hydrogen peroxide. A thiyl radical generated from the reaction between a reductant containing a thiol group and an oxidant was assumed to initiate the polymerization, although the mechanism of the redox reaction was not clear. Later Shukla and Singh ${ }^{2}$ reported the polymerization of acrylamide in aqueous medium using a redox system of thioglycol and potassium bromate.

The author (T.O.) et al. successfully synthesized poly(vinyl alcohol) having a reactive thiol group at an end (PVA-SH). ${ }^{3}$ Using PVA-SH as a reductant and potassium bromate or ammonium persulfate as an oxidant, the aqueous polymerization of acrylic acid, acrylamide and other water soluble monomers that form water soluble polymers was examined. ${ }^{4-6}$ The polymers obtained were assumed to be block copolymers linked to PVA through $-\mathrm{S}-$. Using a similar initiating system, they also carried out the aqueous (co)polymerization of monomers affording water insoluble polymers such as styrene and a mixture of MMA and butyl acrylate (BA). In this case, the block copolymers were formed as emulsions, ${ }^{7,8}$ where the PVA portion in the block copolymers functioned as stabilizer at surfaces of water insoluble polymer particles. However, fractionation of the polymers in these emulsions indicated that the ratio of blocked P(MMA/BA) copolymer with PVA to all of copolymerized MMA/BA was as low as $50-60 \%$. The value might be much higher, if only PVA-S - radical is formed in the redox reaction. The method to synthesize block copolymers by radical mechanism is of importance, since not only non-polar monomers such as styrene but also polar monomers such as acrylamide, acrylic acid, alkyl acrylates and so on which are difficult to blockcopolymerize with ionic mechanism can be utilized as components of the block copolymers. Various types of di-block copolymers will be useful as compatibilizers in polymer application like polymer alloys. Consequently, detailed study of the redox initiation mechanism should be made in relation to the demand to make the efficiency of the block-copolymerization higher. In the preceding paper, the authors reported the polymerization of acrylamide with a redox system of cysteine and potassium bromate, ${ }^{9}$ where cysteine was proved to be a powerful chain transfer agent as well as a component of the redox initiator. In this paper a redox system of cysteine and ammonium persulfate is reported also as a homogeneous model.

\section{EXPERIMENTAL}

\section{Materials}

Acrylamide of EP grade (a product of Nacalai Tesque) was used after recrystallization from methanol solution. L-Cysteine hydrochloride monohydrate (Cys, or cysteine) and ammonium persulfate were of GR grade (Nacalai Tesque) and used as received. Distilled water after deionization was used.

\section{Polymerization Procedure}

Polymerization of acrylamide was carried out as follows: To a $300 \mathrm{~mL}$ round bottomed flask equipped with a nitrogen inlet tube, nitrogen outlet pipe, a glass electrode (to measure $\mathrm{pH}$ ), and a platinum electrode (to measure redox potential), prescribed amounts of acrylamide and $300 \mathrm{~mL}$ water were introduced. After bubbling with nitrogen gas (for about 1 hour) to reach constant potential from anodic one, deaerated aqueous solutions of cysteine and ammonium persulfate were poured in this order. The $\mathrm{pH}$ values were in the range of 4.0 to 4.5 . Conversion was determined iodometrically as reported in the preceding paper. ${ }^{9}$

\section{Molecular Weight Measurement}

By GPC method (Tosoh GPC CPM-II, refractive index detector) using standard aqueous solutions of polyethylene glycol, the degree of polymerization of poly- 
acrylamide was determined.

\section{RESULTS AND DISCUSSION}

Relation between rate of polymerization of acrylamide and concentrations of the redox initiator components was investigated at constant monomer concentration. In Figure 1 are shown time-conversion curves at various concentrations of cysteine and of ammonium persulfate. In Table I are summarized the rate of polymerization obtained in Figure 1, together with number average degree of polymerization $\left(\bar{P}_{n}\right)$ and ratios of weight average to number average degrees of polymerization of polyacrylamide obtained at final conversion in each experiment. Figure 2 shows logarithmic plots of the rate of polymerization against the concentration of cysteine and of ammonium persulfate. From Figure 2, following equation was obtained.

$$
R_{\mathrm{p}}=K[\mathrm{Cys}]^{0.42}[\mathrm{APS}]^{0.55}
$$

The rate of polymerization can be regarded as approximately proportional to the square root of the concentration of each component of the initiator. This means that the redox combination of cysteine and ammonium persulfate is a typical initiator system in free radical polymerization.

Number average degree of polymerization seemed to be affected not only by the rate of polymerization but also concentrations of the redox initiator components.

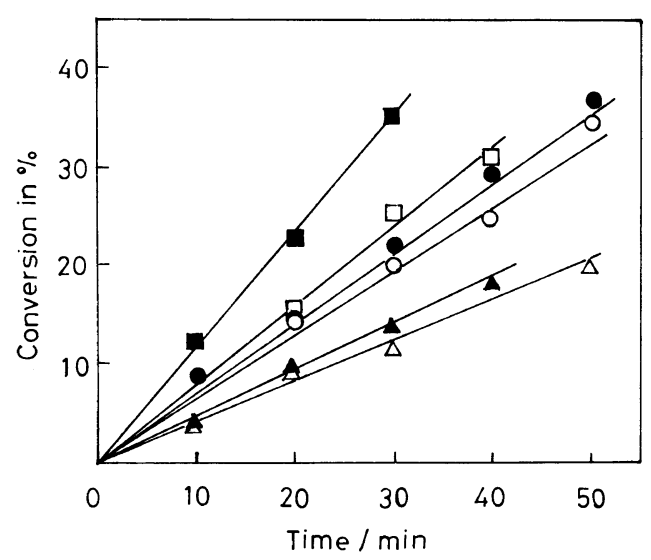

Figure 1. Time-conversion curves at various concentrations of redox components $10^{5}$ [cysteine] $/ 10^{5}$ [APS], in $\operatorname{mol~L}^{-1}: \triangle, 0.50 / 5.0 ; \bigcirc, 1.0 /$ $5.0 ; \square, 2.0 / 5.0 ; \boldsymbol{\Delta}, 5.0 / 1.0 ; \bigcirc, 5.0 / 2.0 ; \square, 5.0 / 5.0$; [monomer], $0.3 \mathrm{~mol} \mathrm{~L}^{-1}$; temperature, $30^{\circ} \mathrm{C}$.

Table I. Rate of polymerization of acrylamide and degree of polymerization of the polyacrylamide at various concentrations of cysteine and ammonium persulfate ${ }^{\mathrm{a}}$

\begin{tabular}{|c|c|c|c|c|c|}
\hline \multirow{2}{*}{$\frac{10^{5}[\mathrm{Cys}]}{\mathrm{mol} \mathrm{L}^{-1}}$} & \multirow{2}{*}{$\frac{10^{5}[\mathrm{APS}]}{\mathrm{mol} \mathrm{L}^{-1}}$} & \multicolumn{2}{|r|}{$R_{\mathrm{p}}$} & \multirow{2}{*}{$\bar{P}_{n}$} & \multirow{2}{*}{$\bar{P}_{w} / \bar{P}_{n}$} \\
\hline & & $\% \min ^{-1}$ & $10^{-5} \mathrm{~mol} \mathrm{~L}^{-1} \mathrm{~s}^{-1}$ & & \\
\hline 5 & 1 & 0.48 & 2.40 & 6180 & 1.90 \\
\hline 5 & 2 & 0.80 & 4.00 & 5480 & 2.20 \\
\hline 5 & 5 & 1.17 & 5.85 & 3020 & 1.94 \\
\hline 0.5 & 5 & 0.39 & 1.95 & 12680 & 2.15 \\
\hline 1 & 5 & 0.66 & 3.30 & 6070 & 2.79 \\
\hline 2 & 5 & 0.80 & 4.00 & 4420 & 2.40 \\
\hline
\end{tabular}

${ }^{\text {a }}$ Concentration of monomer, $0.3 \mathrm{~mol} \mathrm{~L}^{-1}$; temperature, $30^{\circ} \mathrm{C}$.
This will be discussed later in relation to chain transfer reactions to these initiator components. Molecular weight distribution, shown in Table I as the ratio of weight average to number average degrees of polymerization, indicates the polymerization as a typical homogeneous radical polymerization system, since the ratios are approximately 2 or little larger.

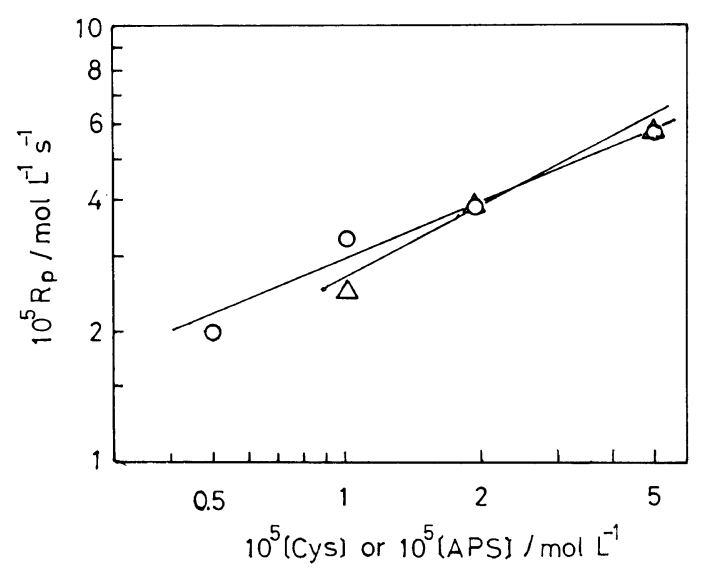

Figure 2. Relation between $R_{\mathrm{p}}$ and [cysteine] or [ammonium persulfate]: $O$, [ammonium persulfate] $5 \times 10^{-5} \mathrm{~mol} \mathrm{~L}^{-1}, \triangle$, [cysteine] $5 \times 10^{-5} \mathrm{~mol} \mathrm{~L}^{-1}$

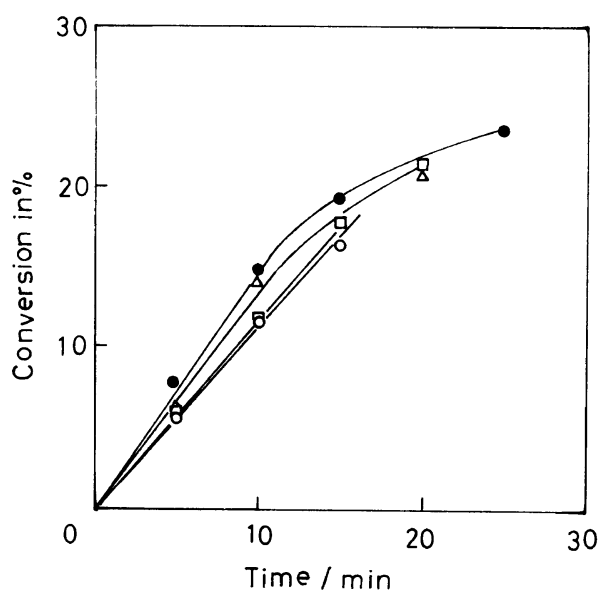

Figure 3. Time-conversion curves at various monomer concentration: [cysteine], $1.0 \times 10^{-5} \mathrm{~mol} \mathrm{~L}^{-1}$; [ammonium persulfate], $5.0 \times$ $10^{-5} \mathrm{~mol} \mathrm{~L}^{-1} ;$ [monomer], in mol L ${ }^{-1} ; \square, 0.2 ; \triangle, 0.3 ; \bigcirc, 0.4 ; \bigcirc, 0.5$; temperature, $30^{\circ} \mathrm{C}$.

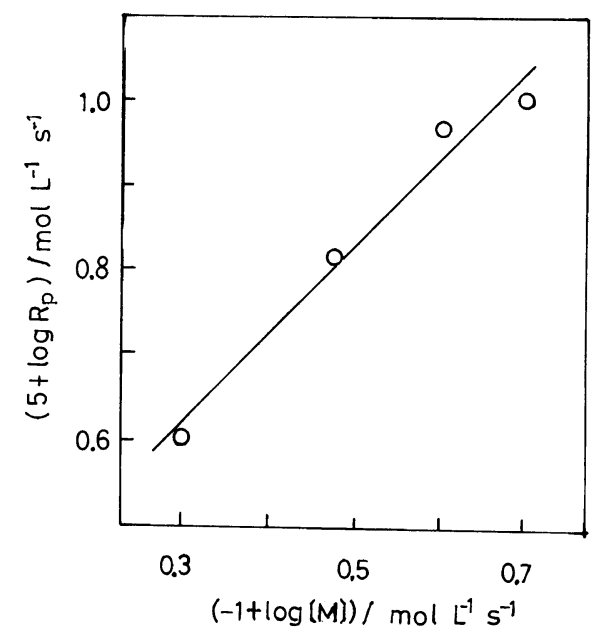

Figure 4. Relation between $\log R_{\mathrm{p}}$ and $\log$ [monomer].

Polym. J., Vol. 29, No. 6, 1997 


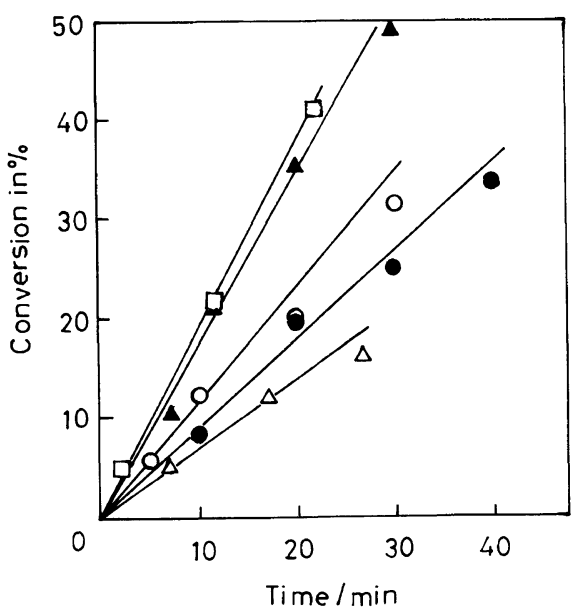

Figure 5. Effects of polymerization temperature on rate of polymerization: [monomer], 0.3; [cysteine], $5 \times 10^{-5}$; [ammonium persulfate], $5 \times 10^{-5}$ in $\mathrm{mol} \mathrm{L}^{-1}$; temperature, in ${ }^{\circ} \mathrm{C} ; \triangle, 20 ; \mathbf{O}, 25 ; \mathrm{O}$, $30 ; \mathbf{\Delta}, 40 ; \square, 50$

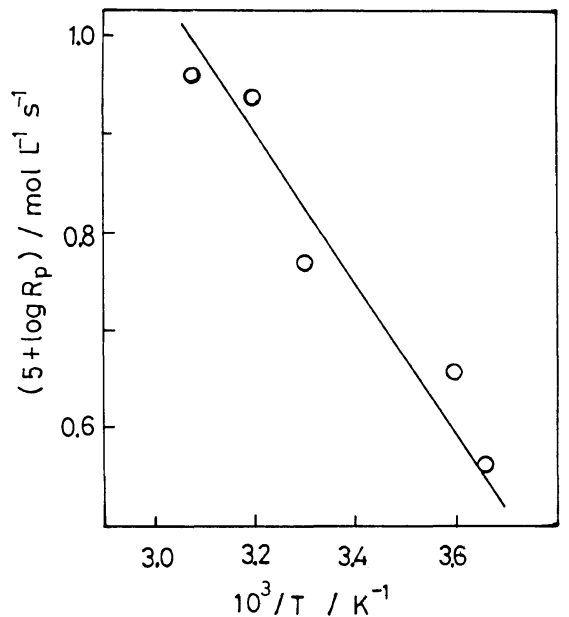

Figure 6. Arrhenius plots of rate of polymerization: Conditions: the same as in Figure 5.

The effects of monomer concentration in $0.2-0.5 \mathrm{~mol}$ $\mathrm{L}^{-1}$ on the rate of polymerization were investigated. Figure 3 shows the time-conversion curves. In Figure 4 are shown logarithmic plots of the obtained rate of polymerization and monomer concentration. From Figure 4 the rate of polymerization was revealed to be proportional to the 1.0 power of the monomer concentration. This power is normal in free radical polymerization in contrast to power 1.8 reported for the polymerization of acrylamide initiated with cysteine and potassium bromate. ${ }^{9}$

The effects of polymerization temperature on the rate of polymerization were investigated. Figures 5 and 6 show time-conversion curves of the polymerization at $20-50^{\circ} \mathrm{C}$ and Arrhenius plots of the rate of polymerization, respectively. From Figure 6 the activation energy of over-all rate of polymerization was obtained to be $4 \mathrm{kcal} \mathrm{mol}^{-1}$. The low over-all activation energy of $4 \mathrm{kcal} \mathrm{mol}^{-1}$ indicates that the system of cysteine and ammonium persulfate is the typical redox initiator; that is, the activation energy of the reaction between both compounds is close to $0 \mathrm{kcal} \mathrm{mol}^{-1}$.

From the results obtained above, the polymerization
Table II. $k$ determined from eq $1^{a}$

\begin{tabular}{|c|c|c|c|}
\hline $10^{5}[\mathrm{Cys}]$ & $10^{5}[\mathrm{APS}]$ & $R_{\mathrm{p}}$ & $k$ \\
\hline $\mathrm{mol} \mathrm{L}^{-1}$ & $\operatorname{mol~L}{ }^{-1}$ & $10^{-5} \mathrm{~mol} \mathrm{~L}^{-1} \mathrm{~s}^{-1}$ & $\mathrm{Lmol}^{-1} \mathrm{~s}^{-1}$ \\
\hline 5 & 1 & 2.40 & 1.28 \\
\hline 5 & 2 & 4.00 & 1.76 \\
\hline 5 & 5 & 5.85 & 1.52 \\
\hline 0.5 & 5 & 1.95 & 1.69 \\
\hline 1 & 5 & 3.30 & 2.42 \\
\hline \multirow[t]{2}{*}{2} & 5 & 4.00 & 1.78 \\
\hline & & & Av. 1.7 \\
\hline
\end{tabular}

${ }^{\text {a }}$ Data: same as in Table I.

initiated with cysteine and ammonium persulfate is regarded to proceed as follows:

$$
\begin{gathered}
\left(\mathrm{NH}_{4}\right)_{2} \mathrm{~S}_{2} \mathrm{O}_{8}+\mathrm{RSH} \stackrel{k}{\longrightarrow} \mathrm{SO}_{4}^{-} \cdot+\mathrm{RS} \cdot+\mathrm{SO}_{4}^{2-} \\
\mathrm{RS} \cdot+\mathrm{M} \stackrel{k_{\mathrm{i} 1}}{\longrightarrow} \mathrm{P} . \\
\mathrm{SO}_{4}^{-} \cdot+\mathrm{M} \stackrel{k_{\mathrm{i} 2}}{\longrightarrow} \mathrm{P} . \\
\mathrm{P} \cdot+\mathrm{M} \stackrel{k_{\mathrm{p}}}{\rightarrow} \mathrm{P} . \\
\mathrm{P} \cdot+\mathrm{P} \cdot \stackrel{k_{\mathrm{t}}}{\longrightarrow} 2 \mathrm{P} \text { or } \mathrm{P}_{2}
\end{gathered}
$$

where $\mathrm{R}$ and $\mathrm{M}$ denote $\mathrm{HOOCCH}\left(\mathrm{NH}_{2}\right) \mathrm{CH}_{2}$ and the monomer, respectively, $k$ is rate constant of the redox reaction, $k_{\mathrm{i} 1}$ and $k_{\mathrm{i} 2}$ are rate constants of initiation reactions, $k_{\mathrm{p}}$ and $k_{\mathrm{t}}$ are rate constants of propagation and termination, respectively. Since the rate of polymerization, $R_{\mathrm{p}}$, is proportional to the square root of concentrations of cysteine and of ammonium persulfate and to the first order of monomer concentration, $R_{\mathrm{p}}$ is expressed as eq 1 :

$$
R_{\mathrm{p}}=\{k[\mathrm{Cys}][\mathrm{APS}]\}^{1 / 2} k_{\mathrm{p}} \cdot k_{\mathrm{t}}^{-1 / 2}[\mathrm{M}]
$$

By adopting published values ${ }^{10}$ of $k_{\mathrm{p}}\left(10^{4} \mathrm{~L} \mathrm{~mol}^{-1} \mathrm{~s}^{-1}\right)$ and $k_{\mathrm{t}}\left(10^{7} \mathrm{~L} \mathrm{~mol}^{-1} \mathrm{~s}^{-1}\right)$ for acrylamide, $k$ was calculated from the eq 1 , and listed in Table II.

As rate constants of typical redox initiator systems such as ferrous ion/hydrogen peroxide and other peroxides, ${ }^{11,12}$ about 50 to $200 \mathrm{~L} \mathrm{~mol}^{-1} \mathrm{~s}^{-1}$ were reported. $1.7 \mathrm{~L} \mathrm{~mol}^{-1} \mathrm{~s}^{-1}$ obtained above is much smaller compared with those of the well-known typical redox initiator systems. But the redox initiator system seems to have considerably higher reactivity than that of cysteine and potassium bromate reported in the preceding paper, ${ }^{9}$ since much higher concentrations of cysteine and potassium bromate compared with those of cysteine and ammonium persulfate were needed to afford the same rate of polymerization; that is, products of the concentrations of former were about 40 times larger than those of the latter. This is not consistent with Maiti and Palit ${ }^{1}$ using MMA as the monomer, who reported potassium bromate to be the most powerful oxidant among those such as potassium persulfate.

In radical polymerization, the number average degree of polymerization $\left(\bar{P}_{n}\right)$ is expressed by the following 
equation:

$$
\frac{1}{\bar{P}_{n}}=C_{\mathrm{m}}+C_{\mathrm{x}} \frac{[\mathrm{Cys}]}{[\mathrm{M}]}+C_{\mathrm{I}} \frac{[\mathrm{APS}]}{[\mathrm{M}]}+\frac{k_{\mathrm{t}} R_{\mathrm{p}}}{2 k_{\mathrm{p}}^{2}[\mathrm{M}]^{2}}
$$

where $C_{\mathrm{m}}, C_{\mathrm{x}}$, and $C_{\mathrm{I}}$ are chain transfer constants to monomer, cysteine, and ammonium persulfate, respectively. In the above polymerizations, where the monomer concentration was varied, the number average degree of polymerization was measured and plotted against polymerization time in Figure 7 . Since the degree of polymerization is dependent on polymerization time (i.e. conversion), values extrapolated to time $0, \bar{P}_{n, 0}$, were used as the number average degree of polymerization. By using the data of the above polymerization and other

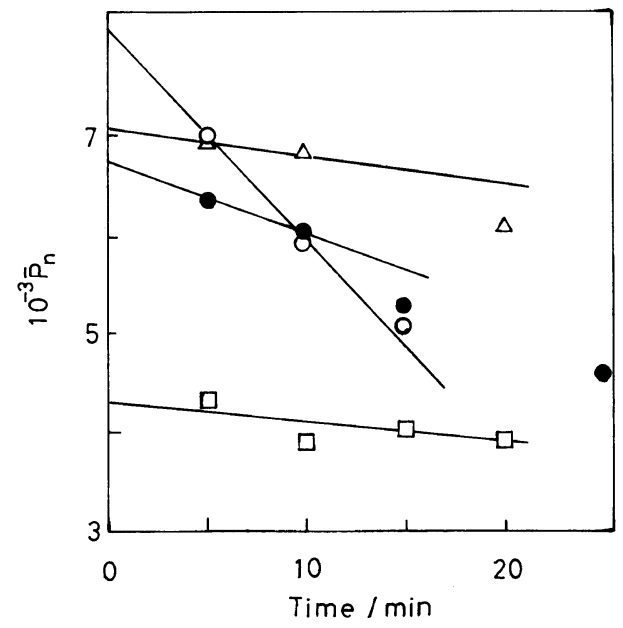

Figure 7. Change in number average degree of polymerization during polymerization: [cysteine], $1.0 \times 10^{-5} \mathrm{~mol} \mathrm{~L}^{-1}$; [ammonium persulfate], $5.0 \times 10^{-5} \mathrm{~mol} \mathrm{~L}^{-1}$; [monomer], in $\mathrm{mol} \mathrm{L}^{-1} ; \square, 0.2 ; \triangle, 0.3$; $0.4 ; 0,0.5$; temperature, $30^{\circ} \mathrm{C}$.

Table III. $C_{\mathrm{I}}^{\mathrm{a}}$ data

\begin{tabular}{cccc}
\hline$[\mathrm{M}]$ & & \multicolumn{1}{c}{$1 / \bar{P}_{n, 0}$} & \\
\cline { 3 - 3 } $\mathrm{mol} \mathrm{L}^{-1}$ & & $\bar{P}_{n, 0}$ & $C_{\mathrm{I}}$ \\
\hline 0.2 & & 23.3 & \\
\hline 0.3 & 4290 & 14.08 & 0.61 \\
0.4 & 7100 & 14.93 & 0.40 \\
0.5 & 6700 & 12.37 & 0.78 \\
\hline
\end{tabular}

Av. 0.65

${ }^{\text {a }}$ Experimental conditions: same as in Figure 7. published $k_{\mathrm{p}}, k_{\mathrm{t}}^{10}$ along with $C_{\mathrm{x}}$ value reported previously as $0.4^{9}, C_{\mathrm{I}}$ was obtained using eq 2 . Table III shows $C_{\mathrm{I}}$. Average value of $C_{\mathrm{I}}$ of 0.65 is rather high. To our knowledge, chain transfer constant data of the growing chain radical of polyacrylamide to persulfates have not been published.

Table IV indicates contribution of each term of right hand side in eq 2 , or the contribution of each term to determine the degree of polymerization. As is clear in the Table, the degree of polymerization was slightly affected by rate of polymerization (about 10\%), and determined mainly with chain transfer reactions to cysteine and ammonium persulfate. The contribution of chain transfer to monomer was about $10 \%$ or less. Since both chain transfer constants to cysteine and ammonium persulfate are large and very close to each other $(0.40$ and 0.65 , respectively), either component at much higher concentration determines the degree of polymerization. For chain transfer to cysteine, RS - is generated, while for ammonium persulfate, $\mathrm{SO}_{4}^{-} \cdot$ is generated as follows:

$$
\begin{gathered}
\text { PAAm } \cdot+\mathrm{RSH} \rightarrow \mathrm{RS} \cdot+\text { PAAm } \\
\mathrm{PAAm} \cdot+\mathrm{APS} \rightarrow \mathrm{SO}_{4}^{-} \cdot+\text { PAAm-SO } \\
\hline
\end{gathered}
$$

When polymers with a thiol end group were utilized as a component of a redox initiator instead of cysteine, the other monomers were polymerized to block copolymers, and new homopolymers were simultaneously formed. ${ }^{4-8}$ In the emulsion polymerization of styrene using polyvinyl alcohol with a thiol end group as a polymer colloid, the thiol end group served as initiator in combination with ammonium persulfate. ${ }^{7}$ In this case, the block copolymerization of styrene to polyvinyl alcohol took place through -S-linkage, but block-efficiency (block-copolymerized styrene to total polymerized styrene) depended on the addition method of ammonium persulfate: the incremental addition of ammonium persulfate afforded higher block-efficiency compared to batchwise addition. The fact can be realized with the discussion described above. The incremental addition, where concentration of the thiol group was much higher than that of ammonium persulfate, must have made the chain transfer to ammonium persulfate much less, resulting in decreased homopolymer forma-

\begin{tabular}{|c|c|c|c|c|c|c|c|}
\hline \multirow{3}{*}{$\frac{10^{5}[\mathrm{Cys}]}{\mathrm{mol} \mathrm{L}^{-1}}$} & \multirow{3}{*}{$\frac{10^{5}[\mathrm{APS}]}{\mathrm{mol} \mathrm{L}^{-1}}$} & \multirow{3}{*}{$\bar{P}_{n}^{\mathrm{a}}$} & \multicolumn{5}{|c|}{ Right hand side of eq $2 \times 10^{5 \mathrm{~b}}$} \\
\hline & & & \multirow[b]{2}{*}{$C_{\mathrm{m}}$} & \multirow{2}{*}{$C_{\mathrm{x}} \frac{[\text { Cys }]}{[\mathrm{M}]}$} & \multirow{2}{*}{$C_{\mathrm{I}} \frac{[\mathrm{APS}]}{[\mathrm{M}]}$} & \multirow{2}{*}{$\frac{k_{\mathrm{t}} R_{\mathrm{p}}}{2 k_{\mathrm{p}}^{2}[\mathrm{M}]^{2}}$} & \multirow[b]{2}{*}{ Total $^{-1}$} \\
\hline & & & & & & & \\
\hline 5 & 1 & 6180 & $1.2(11)$ & $6.7(59)$ & $2.2(19)$ & $1.3(11)$ & 8770 \\
\hline 5 & 2 & 5480 & $1.2(8)$ & $6.7(47)$ & $4.3(30)$ & $2.2(15)$ & 6940 \\
\hline 5 & 5 & 3020 & $1.2(5)$ & $6.7(30)$ & $10.8(49)$ & $3.3(15)$ & 4550 \\
\hline 0.5 & 5 & 12680 & $1.2(9)$ & $0.7(5)$ & $10.8(78)$ & $1.1(8)$ & 7250 \\
\hline 1 & 5 & 6070 & $1.2(8)$ & $1.3(7)$ & $10.8(72)$ & $1.8(12)$ & 6620 \\
\hline 2 & 5 & 4420 & $1.2(7)$ & $2.7(16)$ & $10.8(64)$ & $2.2(13)$ & 5920 \\
\hline
\end{tabular}
tion.

Table IV. Contribution of each term of right hand side in eq 2

${ }^{a}$ Observed. ${ }^{b}$ Numbers in parenthesis denote contribution to total, in $\%$. 


\section{REFERENCES}

1. S. Maiti and S. R. Palit, J. Polym. Sci. A-1, 9, 253 (1971).

2. J. S. Shkula and K. Singh, J. Polym. Sci., Polym. Chem. Ed., 17, 531 (1979).

3. T. Sato and T. Okaya, Makromol. Chem., 195, 163 (1993).

4. T. Sato and T. Okaya, Makromol. Chem., 195, 175 (1993).

5. K. Terada, T. Sato, H. Maruyama, J. Yamauchi, and T. Okaya, Kobunshi Ronbunshu, 49, 885 (1992).

6. T. Okaya and T. Sato, in "Polyvinyl Alcohol-Developments," C. A. Finch, Ed., John Wiley \& Sons, Chichester, U. K., 1992, Chapter 4.
7. K. Yuki, T. Sato, and T. Okaya, J. Polym. Sci., Polym. Chem. Ed., accepted for publication.

8. K. Yuki, M. Nakamae, T. Sato, H. Maruyama, and T. Okaya, submitted.

9. T. Okaya, K. Kikuchi, and Y. Morii, Macromol. Chem. Phys., accepted for publication.

10. K. C. Berger and G. Meyerhoff, in "Polymer Handbook," 3rd ed, J. Brandrup and E. H. Immergut, Ed., John Wiley \& Sons, New York, N.Y., 1989, p II/67.

11. W. L. Reynolds and R. Lumry, J. Chem. Phys., 23, 2560 (1955).

12. S. Hasegawa, N. Nishimura, S. Mitsumoto, and K. Yokohama, Bull. Chem. Soc. Jpn., 36, 522 (1963). 